If this particle had the mass $m_{0}$ and the charge $-e$ of an electron, the radiation damping of its circular motion would be so great as to stop it almost immediately. Mathisson's attempts to establish a connexion between the Theory of Relativity and quantum mechanics would then obviously encounter unsur. mountable difficulties. In the course of a private discussion, Dr. Mathisson expressed, therefore, the view that not only the mass, charge and spin of the particle but also its magnetic moment should be taken into account. It is to be expected, then, that the electronic constants might be chosen in such a way that a free electron would not emit any radiation when moving in the above-mentioned manner. Mathisson is now completing his theory on these lines.

It is possible to show in a very simple 'classical' way that in some particular cases the superimposed action of a magnetic dipole can annihilate the radia. tion of an electric charge in motion. It is well known that when a magnetic dipole with a moment $q$ moves with a velocity $v$, an electric moment

$$
\mathbf{p}=\frac{1}{c}[\mathbf{v q}]
$$

appears. Suppose such a magnetic dipole carrying also an electric charge $\rightarrow e$ moves uniformly around a circular orbit of radius $r$, the frequency being $\nu$ and the dipole axis remaining perpendicular to the plane of the circle, the electric moment (1) will then be directed along the radius vector of the particle. If we put

$$
p=e r
$$

and assume that the sense of motion is such as to produce a magnetic moment opposite to $\mathbf{q}$, the action of the resulting electric moment will be equivalent, at sufficiently distant points, to that of a charge $-e$ in the centre of the circle and a charge $+e$ on the particle. The moving electron charge will be compensated and there will remain only a charge $-e$ at rest in the centre of the circle. At distances large enough there will remain thus only an electrostatic field and the radiation will disappear. Putting $\mathbf{q}=\frac{h}{4 \pi} \frac{e}{m_{0} c}$ and $\mathbf{v}=2 \pi v r$, we get from (1) and

$$
\nu=2 m_{0} c^{2} / h \text {. }
$$

Curiously enough, this is also the frequency of Schrödinger's Zitterbewegung for a Dirac electron in slow motion.

The above considerations seem to me suggestive, though-or rather because-they are based on such crude 'classical' notions.

Institute of Theoretical Physics,

J. W. WEYSSENHOFF.

Jagellonian University,

Cracow. Jan. 15.

${ }^{1}$ Acta physica Polonica, 6, 163 (1937) and 6, 218 (1937).

Coupling between the Orbital and the Spin Angular Momenta of Paramagnetic Ions from Magnetic Measurements

IN a salt of the iron group, though the spin angular momentum of the paramagnetic ion is not directly affected by the crystalline electric fields acting on the ion, yet indirectly it is, owing to its coupling with the orbital angular momentum of the ion, which is easily affected by these fields. The magnetic aniso. tropy of the crystal, and the deviations of the temperature dependence of its principal susceptibilities from the Curie law, are the results of such an indirect influence of the crystal fields on the spin angular momentum exerted through the spin-orbit coupling ${ }^{1}$, and should be the greater the stronger the coupling. Conversely, from the observed anisotropy or the deviations from the Curie law, it should be possible to calculate the strength of the spin-orbit coupling in the ion.

Such a calculation is simple for a nickel salt, and the necessary theoretical expressions have been worked out by Penney and Schlapp ${ }^{2}$. The magnetic anisotropy $\Delta \%$ for any given plane in the crystal, that is, the difference between the maximum and the minimum susceptibilities in the plane, per gram molecule, is given by the relation

$$
\Delta \chi=\frac{8 N \beta^{2}}{3 k T}\left(8 \lambda-\frac{2 \lambda^{2}}{k T}-3 k T\right) \cdot \Delta \alpha,
$$

where $\lambda$ is the constant of spin-orbit coupling, $\beta$ is the Bohr magneton, and $N, k$ and $T$ have their usual significance. The factor $\Delta \alpha$ depends on the crystal fields acting on the $\mathrm{Ni}++$ ions, and on the orientation of the selected plane in the ciystal. Because of the small coefficient of thermal expansion of the crystal, $\Delta \alpha$ will be almost independent of temperature. From measurements on $\Delta \chi$ at two different temperatures it should therefore be possible to eliminate $\Delta \alpha$ and determine $\lambda$.

Using the method described by us in some recent papers ${ }^{3}$, we have measured the magnetic anisotropies of the tetragonal crystals $\mathrm{NiSO}_{4} \cdot 6 \mathrm{H}_{2} \mathrm{O}$ and $\mathrm{NiSeO}_{4} \cdot 6 \mathrm{H}_{2} \mathrm{O}$, at the temperature of liquid oxygen and at room temperature. For both the crystals the anisotropy at $-183^{\circ} \mathrm{C}$. is found to be about $4 \cdot 8_{0}$ times that at $23^{\circ} \mathrm{C}$. From this ratio we obtain for the constant of spin-orbit coupling in $\mathrm{Ni}++$ the value $\lambda=-331 \mathrm{~cm}^{-1}$, which agrees well with the value - $335 \mathrm{~cm} .^{-1}$ obtained from spectroscopic data 4
210 Bowbazar Street,
Calcutta.
Jan. 12.

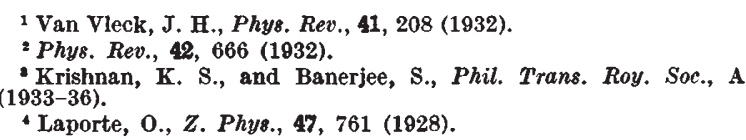

K. S. KRIShNAN.

\section{Diffraction Gratings ruled in Metallic Films}

Prof. R. W. Wood, of Johns Hopkins University, has recently reported ${ }^{1}$ on the successful preparation of diffraction gratings by ruling on 'aluminized' surfaces, and predicts a bright future for this type of dispersive instrument. One of the most interesting aspects of this technique, however, is not included in his letter. Inasmuch as I participated in the conception and development of the general method of preparing and ruling evaporated films at Cornell University early in 1934, which method Prof. Wood was induced to adopt about a year later, I feel that I am in a position to offer some comments of interest.

Diffraction gratings are not ruled upon 'aluminized' mirrors but rather upon mirrors the surfaces of which are coated with a discrete film of aluminium deposited over a film of chromium. It was found in the early attempts to rule gratings in evaporated films of pure aluminium that the aluminium film was stripped in patches from the glass by the diamond point, while films of pure chromium were so hard that a diamond loaded heavily enough to rule the chromium also 